\title{
Construction of Sichuan Liquor Industry Competitiveness Evaluation Model from the Perspective of Diamond Theory
}

\author{
Yuhui Li*, Yijun Chen \\ Sichuan University of Science \& Engineering, Zigong, China \\ Email: ^1037811135@qq.com
}

How to cite this paper: Li, Y. H., \& Chen, Y. J. (2020). Construction of Sichuan Liquor Industry Competitiveness Evaluation Model from the Perspective of Diamond Theory. Journal of Financial Risk Management, 9, 141-154.

https://doi.org/10.4236/jfrm.2020.92008

Received: May 22, 2020

Accepted: June 26, 2020

Published: June 29, 2020

Copyright $\odot 2020$ by author(s) and Scientific Research Publishing Inc. This work is licensed under the Creative Commons Attribution International License (CC BY 4.0).

http://creativecommons.org/licenses/by/4.0/

\begin{abstract}
With the acceleration of economic integration, the development of Internet platform, big data technology, and the elimination of "liquor production line" from the restricted category, the development of Sichuan liquor industry is bound to usher in a new trend. This paper analyzes the current situation of Sichuan liquor industry development, and then based on Porter diamond theory, analyzes the competitiveness of Sichuan liquor industry, on this basis, combined with the Haken synergy theory, constructs an evaluation model to measure the competitiveness of Sichuan liquor industry. This will lay a foundation for the evaluation of the competitiveness of Sichuan liquor industry and the path to enhance the competitiveness of Sichuan liquor industry in the future, so as to promote the high-quality and sustainable development of Sichuan liquor industry. At the same time, it also provides reference for other regions or China's liquor industry competitiveness evaluation.
\end{abstract}

\section{Keywords}

Diamond Model, Harken Synergy Theory, Sichuan Liquor Industry

Competitiveness, Evaluation Model

\section{Introduction}

The ability of different participants to compete for the same resources is called competitiveness by scholars, and competitiveness is studied as a meso-level, that is, comparative industrial productivity, comparative analysis of the advantages of different regions or countries in the same industry (Zhang, 2009). In the early 1990s, China began to carry out relevant research on industrial competitiveness. According to the report on the development of China's international competi- 
tiveness, the industrial international competitiveness consists of six aspects: competitive strength, competitive ability, competitive potential, competitive pressure, competitive power and competitive vitality, which has a significant impact on the research in the field of industrial competitiveness in China (Joint Research Group of the Research Institute of Economic System Reform, Renmin University of China \& China Development Institute, 1997). At present, the most representative concept of industrial competitiveness in China is the comprehensive view put forward by Dr. Jinbei, that is, "industrial competitiveness is the comprehensive quality that a certain industry in one country can provide products or services to the market more effectively than similar industries in other countries" (Jin, 1997). Professor Porter has published many works on industrial competitiveness, elaborated the theory of national competitive advantage, and developed the classic model of evaluating industrial competitiveness- "Diamond Model". Porter believes that the industrial competitiveness is the productivity reflected by the sales of a certain industry in the international market. The industrial competitiveness is formed by the four main factors of production factors, domestic market demand, relevant and supporting industries, enterprise strategic structure and peer competition, as well as two auxiliary factors of government policies and opportunities (Porter, 1990). He believes that these factors should be evaluated separately because they have different impacts on each industry. Since the diamond model was put forward, Porter's theory of industrial competitiveness has far-reaching influence on the academic world. Many scholars use diamond model for reference to study industrial competitiveness.

With regard to the application of diamond model in liquor industry, some scholars have analyzed the current situation and problems of liquor industry development from the qualitative level, and put forward suggestions for liquor industry development (Yang \& Yin, 2015; Wang \& Chen, 2010). However, there is no case of diamond model application in Sichuan liquor industry. Moreover, with the "liquor production line" removed from the restricted category, China's liquor industry is facing new development environment, new opportunities and challenges, and the existing analysis of six factors of diamond model of liquor industry is gradually insufficient. As for the competitiveness of liquor industry, domestic scholars mainly focus on the promotion and evaluation of industrial competitiveness. However, the evaluation system of liquor industry is still in exploration, so there is no final conclusion on the research of Sichuan liquor industry competitiveness, and there is also a lack of research methods on liquor industry competitiveness. Liquor industry is the economic pillar industry of Sichuan Province, which has obvious comparative advantages in the whole country and has an important impact on the economic development of Sichuan Province. Therefore, Sichuan liquor industry urgently needs to make clear the development status of the new stage in order to carry out competitiveness analysis, and urgently needs to determine a new diamond evaluation model with the characteristics of Sichuan liquor industry. 


\section{Basic Theoretical Framework}

\subsection{Diamond Model}

Professor Michael Porter's diamond model theory is based on the industrial competition between countries. It was first put forward in his book "national competitive advantage" in 1900. Porter believes that industrial competitiveness is influenced by four basic determinants: production factors, demand conditions, enterprise factors, related and supporting industries, as well as two auxiliary factors: government and opportunity. The six elements of the diamond model interact to promote or limit the competitive advantage and potential of an industry (see Figure 1).

1) Factors of production. Factors of production can be summed up as natural human resources, capital investment, management resources, scientific information resources, infrastructure conditions, etc. In different industries, the decisive role of production factors on industry is different, and the demand of industry on production factors is also different.

2) Demand conditions. Demand conditions refer to market demand, which is divided into domestic market demand and foreign market demand. Market demand refers to the demand of upstream and downstream of the industrial chain, including demand structure, market scale, consumption capacity, etc. market demand is the driving force of industrial development.

3) Related and supporting industries. This mainly refers to the upstream and downstream industries needed for the development of an industry, such as suppliers, distributors, logistics, etc. Porter thinks that relevant and supporting industries are important factors of industrial competitiveness, because any industry must depend on other relevant and supporting industries, so as to jointly form the competitive advantage of the industry.

4) Enterprise. Enterprises are the commanders of industrial development and play an important role in guiding industrial development. Enterprise factors mainly include enterprise strategy, organizational structure, enterprise competition and cooperation, etc.

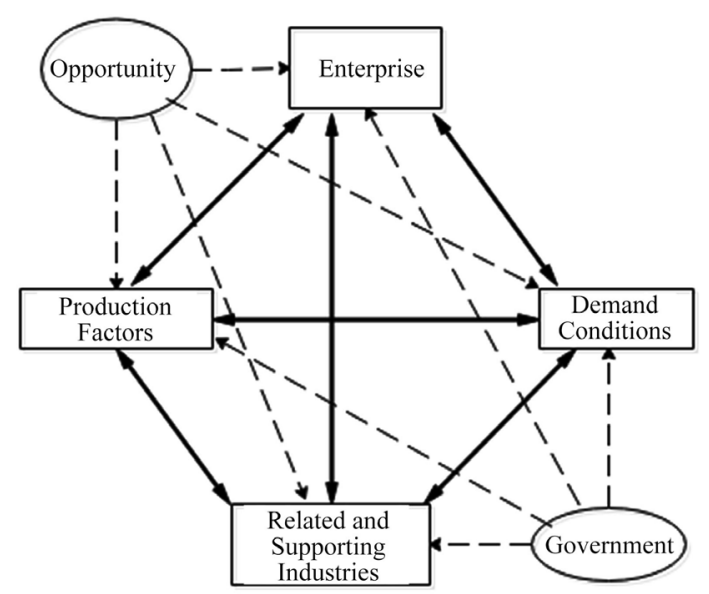

Figure 1. Structure of diamond model (Porter, 1990). 
5) Government. The government's timely encouragement and support will help guide the healthy and orderly development of an industry. The government's formulation of industrial policies and macro-control measures to promote industrial development, as well as the introduction of relevant laws and regulations to stabilize the industrial development environment, plays an important role in the industrial scale structure and growth rate.

6) Opportunity. Opportunity is a variable factor that affects the existing industrial competition pattern. Enterprises in the industry can seize the opportunity to gain competitive advantage by positioning their advantages and disadvantages and taking advantage of the situation.

\subsection{Industrial Competitiveness and Liquor Industry Competitiveness}

As for industrial competitiveness, a relatively mature theoretical system has been formed up to now, such as Porter's "theory of national competitive advantage", Pei Changhong's "theory of comparative advantage and competitive advantage", Jinbei's "view of comprehensive ability", Sheng Shihao's "theory of comprehensive production ability", Zhang Chao's "theory of efficiency ability innovation" and "view of profit" (Liu \& Ouyang, 2003). Based on the existing research results, this study defines "liquor industry competitiveness" as: a country or a certain place liquor industry has different comparative competitive advantages from other economic entities in the market competition, the competitors who are hard to copy in meeting the market demand, profitability, self-development, etc., provide high-quality products and services for the market, and continuously obtain revenue Comprehensive ability of benefits.

\subsection{Current Situation of Sichuan Liquor Industry}

Sichuan liquor, represented by Chengdu, Deyang, Luzhou, Yibin, Mianyang, Suining and Bazhong, is the leader of Chinese liquor industry. It has obvious comparative advantage in the whole country and plays an important role in Chinese liquor industry. By 2020, according to the data of Sichuan Provincial Department of economy and information technology, the output, sales revenue, tax, profit and other economic indicators of Sichuan liquor industry have achieved growth for many years in a row, among which the output and sales of Sichuan liquor accounted for more than $40 \%$ in China in 2018, and the development of Sichuan liquor industry is in the ascendant. In addition, the development of Sichuan liquor industry has shown a pattern of clustering and diversification. However, through the collection and analysis of relevant literature of $\mathrm{Si}$ chuan liquor industry, it is found that there are still many problems in the development of Sichuan liquor industry: the high concentration of Sichuan liquor brands, the large number of enterprises, especially the rapid rise of the second and third tier brands have laid the foundation for the prosperity of Sichuan liquor, but the industrial structure is uneven (Su, 2017); Luzhou, Yibin, Deyang and Chengdu are the four major liquor production areas in Sichuan, with clus- 
ters among them The effect is weak, many enterprise brands have not formed development cohesion (Zhang, Peng, Wu, \& Fan, 2018); Sichuan liquor industry production and sales accounts for a large proportion in the country, but the profit is lower than Guizhou liquor production area, and the added value of liquor needs to be improved (Wang \& Yin, 2018); liquor industry competition is fierce, high-end liquor brands enter the second and third line liquor brand market, and the competition pressure of small and medium-sized enterprises increases and the competition is disordered; liquor enterprises in other provinces' production areas The rapid development of Sichuan liquor has brought regional impact, especially the disadvantage of small and medium-sized enterprises of Sichuan liquor (Yang \& Xu, 2017); the total output and income of Sichuan liquor ranks first in China, but the export is weak, and the international competitiveness is weak (Cheng \& You, 2019). All of these have seriously affected the competitiveness of Sichuan liquor industry.

\subsection{Analysis of Sichuan Liquor Industry Competitiveness}

\subsubsection{Production Factors}

Sichuan Province has a long history of brewing, and has natural brewing conditions: abundant water resources, pure water quality; rich soil minerals, providing support for the healthy cultivation of microbiota; developed agriculture, rich in rice, wheat, corn, waxy sorghum and other food crops, laying a solid foundation for the brewing of liquor. At the same time, due to the fact that many liquor enterprises are built along the river and pay attention to the quality of water sources, not excluding environmental pollution, it will cause a devastating group attack on liquor enterprises, such as Wuliangye plant site built along the Minjiang River, Luzhou Laojiao liquor base located along the Yangtze River, Shuijingfang located along the Jinjiang River, Langjiu located along the Chishui River, etc. Therefore, the liquor industry should timely monitor and do a good job in backup measures, assume social responsibility, upgrade and optimize production technology. In terms of financing, Sichuan liquor industry has established a strong industrial development financing platform, with Wuliangye, Luzhou Laojiao, Tuopai and other famous liquor enterprises well financed; Yibin and Luzhou have liquor development funds. In addition, Luzhou Laojiao, Wuliangye and Jiannanchun are shareholders of Huaxi Securities Company. Luzhou Laojiao has established Longma Xingda small loan company, which provides guarantee for capital supply of liquor industry chain. In terms of technical knowledge and conditions, Sichuan people are proficient in many ways of brewing under the accumulation of historical experience, and some ancient brewing techniques are also listed in the national intangible cultural heritage list. In addition, Sichuan Province has established the liquor industry production technology innovation alliance, and the liquor "production, learning and research" has been on the rise. In Deyang, Chengdu, Yibin, Mianyang and other cities, a number of liquor industry parks have been set up, and a number of well-known liquor enterprises have set up research institutes, allocated special funds to build a production, 
learning and research platform, and jointly promote liquor by virtue of the scientific research advantages, scientific and technological achievements, and educational resources of colleges and universities Innovation of brewing technology, strengthening the scientific research strength and achievement transformation ability of wine enterprises. In terms of labor force, Sichuan wine has always attached great importance to the construction of enterprise talent team. At present, the total number of Sichuan wine practitioners is more than 500,000, with a considerable number of skilled talents, national liquor judges and national liquor brewing masters. These talent advantages provide a human guarantee for Sichuan to create famous and excellent white wine. Sichuan liquor industry also has a number of state-level technical centers, testing centers, state-level liquor laboratories, as well as top-level organizations such as Sichuan China liquor Golden Triangle liquor industry association and Sichuan Liquor Making Research Institute.

\subsubsection{Demand Conditions}

With the upgrading of the national consumption level and the improvement of the consumer income level, people's growing demand for a better life has become one of the main contradictions. Various demands such as healthy drinking, wine culture inheritance and development are increasingly accepted by the public. Chinese people pay more attention to the improvement of the material and cultural quality of life. In addition, the novel coronavirus pneumonia affected the consumption structure of the residents, and from the consumption expenditure point of view (consumer spending, including food, tobacco and liquor, clothing, housing, daily necessities and services, transportation and communications, education, culture and entertainment, health care and other products and services of the eight categories, as shown in Figure 2), the first quarter of 2020, the residents per capita consumption of food, tobacco and alcohol consumption accounted for the largest proportion (33.6\%). According to the data released by the National Bureau of statistics, per capita food, tobacco and alcohol consumption expenditure and per capita residential consumption expenditure are the fastest growing items of consumption expenditure (up $2.1 \%$ ), and the other six items are all down year on year. This shows that there are changes in life style and consumption concept of residents during the epidemic period, but food is the priority of people, and there are still stable consumer groups and demand in the alcohol market, which is difficult to change due to the environment. It is not optimistic that there are serious homogenization and disordered marketing in Sichuan liquor market. Therefore, there is an urgent need to establish a unified and standardized liquor consumption market, to create an advantageous liquor industry cluster production area, to break the regional division; It is necessary to expand the market demand of the second and third line, tap the brand connotation of the second and third line liquor, and spread the traditional culture of liquor. It is also necessary to improve the development policy of liquor industry, so as to establish a good environment for the development of Sichuan liquor industry. 


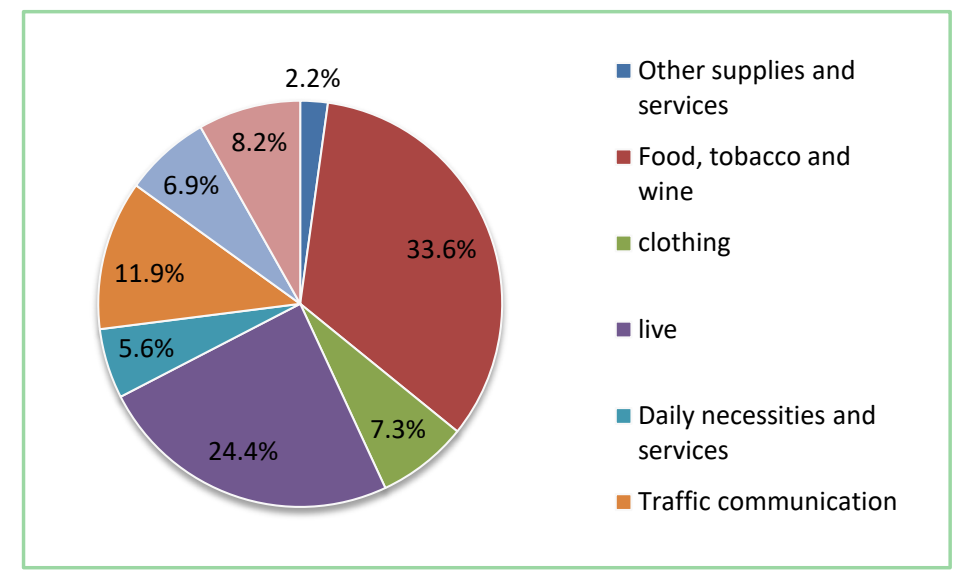

Figure 2. Composition of per capita consumption expenditure in the first quarter of 2020 (data source: National Bureau of Statistics).

\subsubsection{Related and Supporting Industries}

Sichuan liquor industry has a complete and convenient industrial chain division system, including the upstream packaging and printing industry, grain planting industry, the midstream liquor brewing industry, the downstream liquor wholesale retailers and end consumers, from raw material (raw grain, base liquor, packaging materials, production equipment) supply, feed processing, liquor making enterprises, headquarters distributors, logistics, cultural tourism, etc The development trend of supporting industries is good.

Sichuan Province strictly controls the quality of special grains for brewing such as nuohong sorghum, rice and corn, and provides special funds to support the construction of special grain bases for brewing, build special grain bases for brewing, and improve the overall efficiency of special grain production for brewing. It provides special funds to support the construction of special grain bases for brewing, builds special grain bases for brewing in accordance with the standards of modern agricultural parks, and improves the overall efficiency of special grain production for brewing. This ensures the need of Sichuan liquor industry to manufacture high-quality liquor and further improves the competitiveness of Sichuan liquor industry. Most of the packaging and printing enterprises in Sichuan Province are located in Chengdu, Deyang, Mianyang, Yibin and other places, which are also the origin of high-quality liquor. Wuliangye, Jiannanchun, Shuijingfang, Tuopaiqujiu and other large distilleries also have their own printing enterprises, which can produce high-end wine boxes and also undertake external orders. A few products that cannot be produced by themselves or are uncompetitive are outsourced. In addition, Sichuan Province has integrated liquor culture into tourism, and has built a liquor culture tourism route of Chengdu Mianzhu Shehong Luzhou Yibin Chengdu, covering Shuijingfang site of Chengdu, Quanxing Daqu distillery, Mianzhu Jiannanchun distillery, Shehongtuopaiqu distillery, Luzhou Daqu old cellar, Gulinlang distillery, Yibin Wuliangye distillery area, cave type liquor fermentation cellar of Ming Dynasty, Shibin The largest winery in the world (Meng, Guo, Yin, \& Yang, 2014). It plays 
an important role in spreading and carrying forward the traditional liquor culture of Sichuan and even the whole country, building the brand culture of liquor enterprises and increasing the financial revenue.

\subsubsection{Enterprise Factors}

Sichuan liquor industry is similar to a dumbbell structure, that is, "six golden flowers of Sichuan liquor" is the first one, "ten small golden flowers of Sichuan liquor" is the abdomen, and many OEM liquor enterprises, original liquor enterprises and small distilleries throughout Sichuan liquor production area are the tail ones, showing an obvious three-level echelon organization. Among them, six Jinhua, represented by Wuliangye, Luzhou Laojiao, Jiannanchun, Quanxing Shuijingfang, Langjiu and Tuopai Shede, are well-known brands in China, mainly producing medium and high-grade liquor, leading the development of Sichuan liquor industry; ten new Jinhua, represented by Fenggu, Wenjun, Sanxi, Guchuan, Yuanhong Xiaojiaolou, Xufu, Jiangkou alcohol, Xiantan, Guanghan Jinyan and Yuchan Jinxiao Jinhua is the second-line brand of Sichuan white wine industry. It mainly produces medium and low-grade liquor, which is the backbone of Sichuan white wine industry. Famous and excellent liquor represented by "six Jinhua" and "Ten Little Jinhua" is leading the high-quality development of Sichuan liquor industry.

1) Corporate Strategy

With the acceleration of economic integration and the development of Internet big data technology, enterprises pay more and more attention to institutional competitiveness, marketing competitiveness, brand competitiveness, financial competitiveness, brand competitiveness, innovation ability, social responsibility ability and export strategy. The high profit, low barriers to entry and large demand of liquor drive a large number of small and medium-sized enterprises to increase, which further intensifies the internal competition of liquor industry. Throughout the Sichuan liquor brands, Wuliangye of Yibin has entered the ranks of world brands. Luzhou Laojiao and Jiannanchun are national brands, while the rest are trans-provincial or local brands and original liquor. Therefore, the advantages of local brand culture resources in Sichuan liquor industry are not fully explored. At the same time, the strength of small and medium-sized enterprises is not strong, they do not enter the production network, and the agglomeration synergy effect is weak. In terms of brand building, it is faced with the tragedy of the construction site, the external expansion is not suitable, and the internal competition is serious (Yang, Fu, \& Wang, 2019). For the sustainable and high-quality development of Sichuan liquor industry, the liquor enterprises in the industry need to adjust the strategic structure of the enterprise and march forward the road of enterprise modernization.

2) Competition and Cooperation of Enterprises

Competition and cooperation among liquor enterprises will help to enhance the initiative of independent innovation of the whole liquor industry. First class famous liquor enterprises, such as Maotai, Wuliangye and Luzhou Laojiao, have 
established liquor industry development parks one after another, embarked on the road of liquor industry cluster development, expanded production scale and enhanced brand competitive advantage. The golden triangle of Chinese liquor, with Luzhou, Renhuai and Yibin as the top three places, has formed a tripartite liquor industry cluster, which has improved the core competitiveness of Sichuan liquor and Guizhou liquor, and rapidly promoted the influence of liquor at home and abroad. On the whole, the scale economy advantage of Sichuan liquor industry has been formed.

\subsubsection{Government Policies}

The healthy and orderly development of liquor industry is inseparable from the guidance, encouragement and support of the government. From 1984 to now, the ups and downs of the state's policies on liquor industry are also the epitome of the development of liquor industry: in 1998, the state implemented the production license system for the liquor industry to limit the expansion of the production scale of liquor enterprises; in 2001, the state implemented the new policy of "composite tax calculation" for the liquor industry, and cancelled the policy of deductible consumption tax for purchased liquor, which seriously impacted the middle and low-grade liquor industry; in 2006 The state further strictly controls the application for production license of liquor and alcohol production enterprises; in 2011, it issued price limit order to some liquor enterprises. Of course, behind the restrictions lies the fact that the liquor industry is developing rapidly and the problems are exposed. During this period, there is no lack of national policy support for the liquor industry. On January 1, 2020, "liquor production line" will be removed from the restricted category in the guidance catalogue for industrial structure adjustment (2019) edition of the national development and Reform Commission, which will be a major opportunity for the development of liquor industry, and Sichuan, as the characteristic and advantageous production area of Chinese liquor, will usher in a major new trend of development. It can be seen that the government's formulation of industrial policies and macro-control measures to promote the development of the liquor industry, as well as the introduction of relevant laws and regulations to stabilize the liquor industry development environment, plays an important role in the scale structure and growth rate of the liquor industry. Because the industrial policy restricts the establishment of the dominant production areas of liquor industry from the macro level, and the inaccurate standards of liquor industry will directly lead to the inability to effectively guarantee liquor quality and intellectual property rights, which is not conducive to the high-quality sustainable development of liquor industry, nor conducive to promoting China's liquor to the international market.

\subsubsection{External Opportunities}

Liquor is a kind of enjoyment consumer goods, the key is to grasp consumers and tap the cultural resonance in consumers' subconscious. The arrival of Inter- 
net platform and big data era will provide opportunities for online marketing of liquor industry and the spread of brand culture, which will help liquor enterprises to further enhance their corporate cultural image and compete for liquor market share. Meanwhile, one belt, one road strategy has accelerated the export of traditional liquor culture in Sichuan. Sichuan liquor export strategy has become increasingly important in the liquor industry (Yang \& Xu, 2017). But on the other hand, brandy, whisky and vodka also occupy the market share of liquor. There are also some internationally renowned liquor enterprises that merge and acquire China's excellent liquor enterprises, thus directly entering the domestic market. Due to the low entry threshold, high market input, low market risk and high capital return, the excess profit attracts a large number of international capitals to enter the liquor industry in China. The most typical examples are Goldman Sachs' stake in Anhui Kouzijiao wine industry, Diageo's acquisition of Sichuan Shuijingfang wine industry, and Hennessy's acquisition of 55\% of Wenjun wine equity in Jiannanchun. In a word, the safety of Chinese liquor industry should be paid attention to.

\section{Evaluation Model Construction of Sichuan Liquor Industry Competitiveness}

Through the analysis of the competitiveness of Sichuan liquor industry by Porter diamond model, the model is revised to make it more suitable for the actual situation of the development of Sichuan liquor industry, more suitable for evaluating the competitiveness of Sichuan liquor industry, so as to clarify the competitive advantages and disadvantages of Sichuan liquor industry, and put forward countermeasures to enhance the competitiveness.

\subsection{Synergy Theory}

Coordination theory was originally a concept in physics. It was founded by Hermann Haken, a famous physicist. According to Haken, an open system reacts with the external environment in a state of imbalance, and spontaneously realizes the structural ordering of time, space and energy through its internal coordination. The main content of synergetic theory includes three aspects: first, synergetic effect, which is produced by the interaction of many subsystems in an open system; second, servo principle, which is dominated by collective variables (order parameters); third, self-organization principle, which can automatically form certain internal subsystems according to certain rules without the influence of external environment Structure or function, that is to say, a new ordered structure of time, space or function is formed through the cooperation among a large number of subsystems, so it has the characteristics of internality and autogenesis.

\subsection{Evaluation Model}

According to the connotation and main content of synergy theory, this theory 
can be integrated into the development of Sichuan liquor industry, that is, to establish a unique and unrepeatable development system of Sichuan liquor industry. Human intervention makes the liquor industry form a spontaneous operation mechanism and develop in coordination. In case of force majeure or major difficulties, Sichuan liquor industry development system will not fall apart. Therefore, it is necessary to establish an evaluation model suitable for evaluating the competitiveness of Sichuan liquor industry. According to the model, we can evaluate and analyze the competitive advantages or disadvantages, so as to formulate feasible strategies and paths, and take appropriate measures to promote the sustainable and high-quality development of Sichuan liquor industry, and create the world famous liquor-China Liquor-Sichuan liquor (see Figure 3).

As shown in Figure 3, based on the coordination theory of Haken, an evaluation model for the competitiveness of Sichuan liquor industry, namely the new diamond model, which is composed of the endogenous competitiveness subsystem and the exogenous competitiveness subsystem, can be constructed. First of all, endogenous competitiveness is represented by production factors and demand factors, while exogenous competitiveness is represented by industry factors and enterprise factors. Finally, the four elements of endogenous competitiveness and exogenous competitiveness subsystem of this model are the core. Secondly, the model focuses on the composition and synergy of the factors affecting the competitiveness of liquor industry, and gathers the two important factors of policy and opportunity to influence the competitiveness of liquor industry. Each element in the new diamond model plays an important role, which has a significant impact on the overall competitiveness of the industry. At the same time, the six elements interact with each other. Therefore, the evaluation model must pay attention to the cooperation and interaction between the key elements at the same time. It should also be noted that in the Sichuan liquor industry evaluation model, policy and opportunity are no longer auxiliary factors of Porter diamond model, but another important component of liquor industry competitiveness evaluation model. They interact with the four core elements of endogenous competitiveness subsystem and exogenous competitiveness subsystem.

\subsection{Application of Evaluation Model}

The principle of the competitiveness evaluation model of Sichuan liquor industry (new diamond model) is to evaluate the competitiveness of Sichuan liquor industry by analyzing the six factors of endogenous competitiveness (production factors, demand factors) and exogenous competitiveness (industry factors, enterprise factors), as well as policies and opportunities. As for the evaluation method of the new diamond model, the comprehensive evaluation methods such as factor analysis, AHP, fuzzy evaluation, grey correlation analysis and data envelopment analysis can be used to evaluate the industrial competitiveness. 


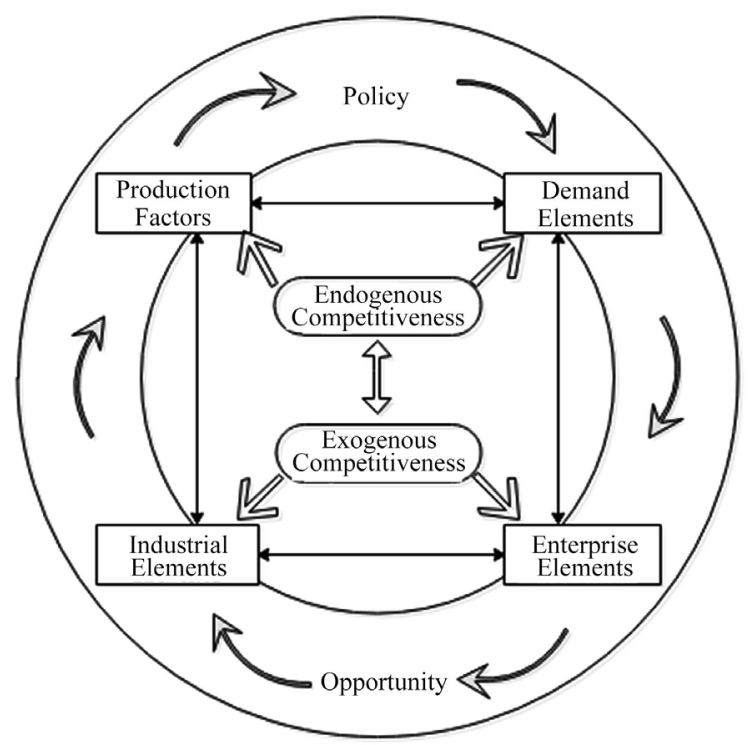

Figure 3. Competitiveness evaluation model of Sichuan liquor industry.

1) In terms of production factors. It can be measured by human resource factors, natural resources, capital, technology, etc., such as liquor practitioners, water resources, forest resources, capital investment, scientific research funds and patent number, etc.

2) In terms of demand factors, it can be analyzed from two dimensions of internal market and external market, and evaluated by market sales and consumer consumption, such as import and export trade, online retail, social market retail, etc. consumer consumption mainly refers to per capita disposable income, per capita network sales, per capita consumption level, etc.

3) Industrial factors. Based on the analysis of resources of related cooperative or supporting industries, it is mainly determined by the situation of stakeholders in the liquor industry chain, such as the resources of special grain planting industry, packaging and printing industry, logistics industry, etc.

4) Enterprise factors. It includes enterprise strategy, industrial structure and enterprise cooperation. It mainly investigates enterprise development strategy and enterprise cluster development plan.

5) Policy. This paper mainly analyzes the influence of government behavior on the development of liquor industry, that is, the policy mechanism and asset allocation of the government on liquor industry. The development environment of liquor industry, liquor quality and Sichuan liquor gold brand can be guaranteed through the government's monitoring and strong standardization.

6) Opportunity. One belt, one road, is the most prosperous area in China. The economy of our country has entered the "new normal", and the electricity supplier market has been booming. Artificial intelligence and big data technology have become the hot research areas in China. The construction of "one belt and one road" has been continuously pushed forward, and China's huge population advantage and strong liquor consumption culture have brought about the op- 
portunities that cannot be missed for the development of Sichuan liquor industry.

Chengdu City, Deyang City, Mianyang City, Suining City, Yibin City, Bazhong City and Luzhou City in Sichuan Province are seven key liquor development cities in response to the call of the provincial government and the opinions on optimizing regional industrial layout, and are the main liquor production areas in Sichuan Province. Through the combination of evaluation model and evaluation method, we will clearly observe the competitive advantages and disadvantages of Sichuan liquor industry, and clearly observe the competitive advantages and disadvantages of each production area of Sichuan liquor, so as to put forward countermeasures to enhance the competitiveness of Sichuan liquor industry from the macro and micro level. At the macro level, from the perspective of the whole Sichuan liquor industry, we are moving towards the direction of cluster development of production areas to build the brand influence of Chinese liquor Sichuan liquor. At the micro level, according to the fact that Sichuan liquor industry is divided into various production areas and based on the liquor market reality, we propose the unique development and optimization path for each liquor production area. Finally, all services are provided for the revitalization of Sichuan liquor industry and the expansion and strength of Chinese liquor enterprises.

\section{Summary}

This paper interprets Porter diamond model, analyzes and combs the current situation of Sichuan liquor industry in view of the new stage and new environment of liquor industry development, and applies diamond model to analyze the competitiveness of Sichuan liquor industry. Based on the analysis of Haken synergy theory, a new competitiveness evaluation model, namely new diamond model, is proposed for Sichuan liquor industry. The biggest innovation of this paper is content innovation, which is to build an evaluation model suitable for evaluating the competitiveness of Sichuan liquor industry. In this evaluation model, government behavior and external opportunity environment become the important factors to measure the competitiveness of liquor industry, not the auxiliary factors in Porter diamond model. The limitation of this paper is not to introduce case analysis to verify the evaluation model, and to propose real-time solutions to the current situation and problems faced by Sichuan liquor industry. The main contribution of this paper is to lay a foundation for the evaluation of the competitiveness of Sichuan liquor industry and the acquisition of the competitiveness promotion path in the future, which can be used as a reference for the next research, and also for the evaluation of the competitiveness of other regions' liquor industry.

\section{Conflicts of Interest}

The authors declare no conflicts of interest regarding the publication of this paper. 


\section{References}

Cheng, T. Y., \& You, Y. H. (2019). Study on the Export Strategy of Sichuan Baijiu. The Food Industry, 40, 249-254.

Jin, B. (1997). China's Industrial International Competitiveness: Theory, Method and Empirical Research. Beijing: Economic Management Press.

Joint Research Group of the Research Institute of Economic System Reform, Renmin University of China, \& China Development Institute (1997). China International Competitiveness Development Report. Beijing: China Renmin University Press.

Liu, X. T., \& Ouyang, K. (2003). A Review of Industrial Competitiveness Research. Contemporary Finance \& Economics, 11, 85-88.

Meng, B., Guo, W. L., Yin, Q. F., \& Yang, L. (2014). Analysis of the Present Situations of Wine Culture-Oriented Tourism in China and Its Development Prospects. Liquor-Making Science \& Technology, 11, 104-110.

Porter, M. E. (1990). The Competitive Advantage of Nations. London: Macmillan. https://doi.org/10.1007/978-1-349-11336-1

$\mathrm{Su}, \mathrm{K}$. (2017). New Growth Path of Liquor Industry in China under the Structural Reform of Supply Side. Journal of Sichuan University of Science \& Engineering (Social Sciences Edition), 32, 14-25.

Wang, L., \& Chen, L. (2010). Analysis of the Competitiveness of Guangxi White Wine Industry Based on Diamond Model. Guangxi Journal of Light Industry, 26, 115-116 + 128.

Wang, Y. Z., \& Yin, B. (2018). Research on the Internationalization of Sichuan Baijiu under the "One Belt and One Road" Initiative. China Brewing, 37, 205-208.

Yang, H., \& Yin, W. H. (2015). Research on Transitional Cluster of Shanxi Xinghua Village Liquor Industry from the Perspective of Diamond Model. World Regional Studies, 24, 138-147.

Yang, L., \& Xu, J. (2017). Construction of Sichuan Baijiu as "National Business Card" under the Background of the Belt and Road Initiative. Liquor-Making Science \& Technology, 9, 112-116.

Yang, L., Fu, L., \& Wang, J. M. (2019). Regional Umbrella Branding of Sichuan Baijiu: Theoretical Logic and Implementation Framework. Liquor-Making Science \& Technology, 8, 132-138.

Zhang, M., Peng, Y. H., Wu, S., \& Fan, L. L. (2018). Research on the Lock-In Effect of Local Industrial Cluster-A Case Study of the Golden Triangle of Chinese Liquor (Sichuan Liquor). Soft Science, 32, 109-112.

Zhang, Y. (2009). Industrial Competitiveness of the Relevant Theory and Its Model Analysis. Economic Research Guide, 15, 15-16. 